\title{
Overall Impairment of Quality of Life in Japanese Patients with Hidradenitis Suppurativa: Comparison with National Standard
}

Koremasa HAYAMA ${ }^{1}$, Hideki FUJITA ${ }^{1}$, Takashi HASHIMOTO ${ }^{2}$ and Tadashi TERUI ${ }^{1}$

${ }^{1}$ Division of Cutaneous Science, Department of Dermatology, Nihon University School of Medicine, 30-1 Oyaguchi-Kamicho, Itabashi-ku, Tokyo 173-8610 and 'Department of Dermatology, Osaka City University Graduate School of Medicine, Osaka, Japan. *E-mail: fujita.hideki@ nihon-u.ac.jp

Accepted Dec 22, 2021; Epub ahead of print Dec 22, 2021

Hidradenitis suppurativa (HS) is a chronic, relapsing, and debilitating cutaneous follicular disease (1). HS markedly affects patients' quality of life (QoL) $(2,3)$, by causing bad odour, contractures, and pain. However, there is little research into how the QoL of HS patients is changed in comparison with the general population.

In a previous study, we found that Japanese patients with HS are characterized by male predominance, frequent involvement of the buttocks, and rare family history (4). The current study aimed to investigate the QoL of Japanese patients with HS, using the skin diseasespecific QoL scale, Dermatology Life Quality Index (DLQI), and Short Form-36 health survey version 2 (SF36v2), a comprehensive health-related QoL scale that is applicable to various types of diseases (5).

\section{MATERIALS, METHODS AND RESULTS}

A questionnaire-based study was performed at 21 institutions in Japan, as described previously in a Japanese HS study (4). Data on patients' age, sex, medical history, family history, duration of the disease, comorbidities, disease severity, and QoL were obtained from April 2018 to March 2020. Disease severity was assessed according to physicians' judgments (Physicians' Global Assessment; PGA: mild, moderate, severe, and very severe), modified Sartorius scores (mSS) (6), and Hurley stage. Japanese-language versions of the DLQI and SF-36v2 $(7,8)$ were used for evalua- tion of QoL. To compare patients' data with those in the general population in Japan, we used the national standard values for Japan in 2017, set out by i-Hope International (Kyoto, Japan). The value of each SF-36v2 element from the national standard population was adjusted to a mean \pm standard deviation (SD) of $50 \pm 10$ points. Then, the values of individual SF-36v2 elements of the patients with HS were converted to obtain T-scores (deviation scores) (9).

The study was approved by the ethics committee of Nihon University Itabashi Hospital (RK- 180313-07). The SF-36v2 license for this study was obtained from i-Hope International (Kyoto, Japan).

Statistical analyses were performed using GraphPad Prism version 8 (GraphPad Software, La Jolla, CA, USA), in which $p<0.05$ was considered statistically significant.

A total of 63 patients' data (49 men and 14 women) were corrected $\left(\right.$ Table $\left.\mathrm{SI}^{1}\right)$. The mean \pm SD age of the patients was $44.4 \pm 11.4$ years. Eleven $(17.5 \%)$ patients had a family history of HS. The mean \pm SD disease duration was $168.7 \pm 135.5$ months. Obesity, diabetes mellitus, hypertension, and hyperlipidaemia were observed in $10(31.7 \%), 12(19.0 \%), 15(23.8 \%)$, and $10(15.9 \%)$ of the patients, respectively. Based on PGA, $16(25.4 \%), 17(27.0 \%)$, $21(33.3 \%)$, and $9(14.3 \%)$ were categorized into mild, moderate, severe, and very severe, respectively. According to Hurley stage classification, $8(12.7 \%), 21(33.3 \%)$, and $34(54.0 \%)$ patients were at the stages of I, II, and III, respectively. The mean \pm SD mSS was $90.11 \pm 95.81$.

The mean \pm SD DLQI of the patients with HS was $9.87 \pm 8.85$. To examine whether the DLQI changed depending on the disease severity assessed by PGA and Hurley stage, Kruskal-Wallis tests
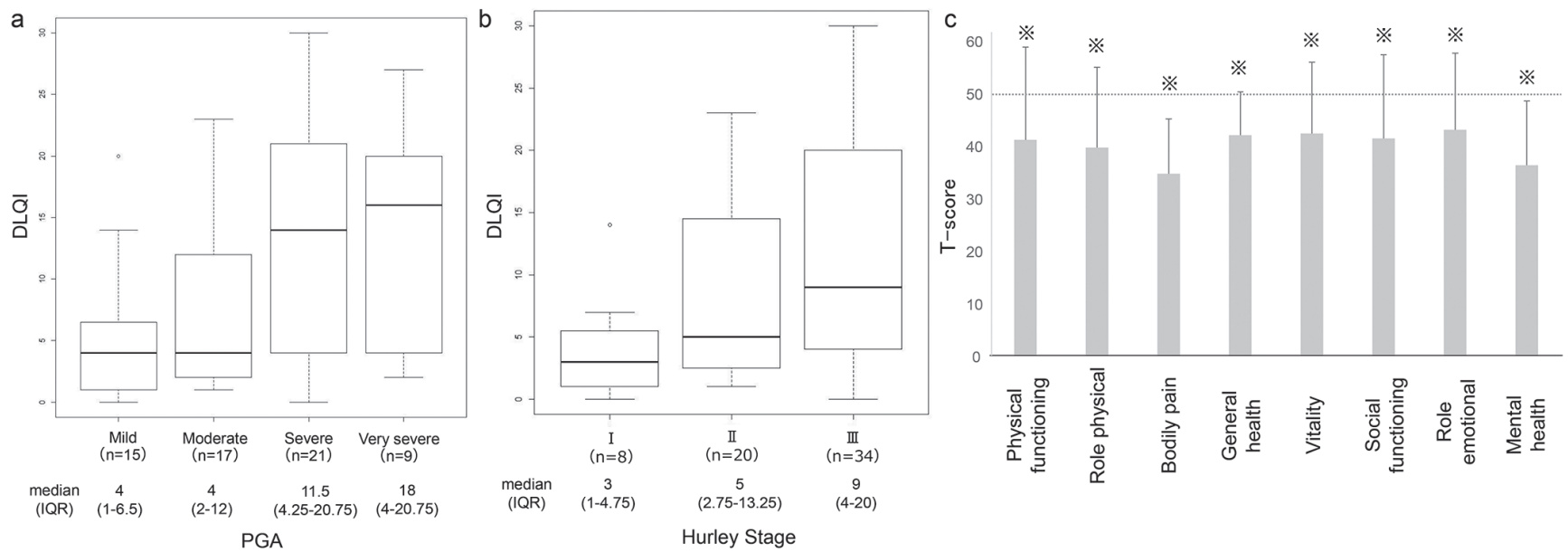

Fig. 1. (a) Correlation between Dermatology Quality of Life Index (DLOI) and Physicians' Global Assessment (PGA). DLOI was significantly correlated with PGA ( $p=0.0189$; Kruskal-Wallis test). (b) Correlation between DLQI and Hurley stage. DLQI was significantly correlated with the Hurley stage $\left(p=0.033\right.$; Kruskal-Wallis test). Within each box, horizontal bold line denote median value. Boxes indicate the range between the $25^{\text {th }}$ and $75^{\text {th }}$ percentiles. IQR: interquartile range. (c) T-scores for the SF-36 v2 elements in patients with hidradenitis suppurativa. T-scores for all the SF-36v2 8 elements were significantly lower in patients with hidradenitis suppurativa compared with Japanese national standard values. $* p<0.05$ (Z-test) Data are shown as means \pm standard deviations. 
were performed as each data showed a non-normal distribution. Significant DLQI differences were demonstrated among each degree of severity in both PGA (Fig. 1a, $p=0.0189$ ) and Hurley stage (Fig. 1b, $p=0.033$ ). We also examined whether DLQI correlated with mSS, using Spearman's rank correlation coefficients, and found a slightly significant correlation between them with a correlation coefficient of 0.393 (Fig. S $1^{1}, p=0.001$ ).

Next, we analysed the general health QoL status obtained by SF-36v2. Each score of SF-36v2 elements was converted to a T-score calculated from national standard value. The T-score for each subscale was as follows: physical functioning: $43.2 \pm 15.4$, role-physical: $42.4 \pm 15.0$, bodily pain: $38.0 \pm 12.9$, general health: $42.0 \pm 9.68$, vitality: $46.1 \pm 12.7$, social functioning: $44.7 \pm 14.1$, role-emotional: $43.3 \pm 15.1$, mental health: $48.0 \pm 12.6$. The T-scores for all 8 subscales were significantly lower in patients than those in the 2017 national standard population (Fig. 1c).

\section{DISCUSSION}

The positive correlation between DLQI and disease severity in the current study is consistent with the results of previous studies $(11,12)$. While mean \pm SD DLQI of patients with HS was $9.87 \pm 8.85$ in this study, recent Japanese research reported that mean DLQI in patients with chronic urticaria, atopic dermatitis, and psoriasis vulgaris were $4.8 \pm 5.1,6.1 \pm 5.5$, and $4.8 \pm 4.9$, respectively (13). Therefore, QoL of patients with HS seems to be more severely impaired than those of other skin diseases.

Unlike DLQI, SF-36v2 can be used for comparison between skin diseases and ailments of other organs (5). Moreover, data from the general populations of various countries are determined, which allow for comparisons with the data for various diseases. Using Japanese standard values, we demonstrated that all 8 elements in SF-36v2 were significantly impaired in patients with HS. To our best knowledge, the current study is the first to compare QoL data of patients with HS with those of the general population using the complete SF-36v2 and to demonstrate a reduction in overall QoL in patients with HS.

The current study has several limitations. First, it was conducted at relatively large hospitals, possibly leading to preferential inclusion of more severely affected patients. Secondly, treatments were not considered. Furthermore, the study was conducted when biologics were unavailable for HS. Thirdly, there is considerable male predominance. Patients with HS in the Far East region show a male predominance (14). Reports from Western countries indicate that female patients with HS have higher DLQI (11). Lack of sex differences in QoL found in this study may be due to the small number of female patients.

In conclusion, this study shows, for the first time, that the QoL of patients with HS is comprehensively impaired compared with the standard population.

\section{ACKNOWLEDGEMENTS}

The authors thank the doctors who cooperated with this research.

This work was supported by Health Labor Sciences Research Grant Number 20FC1026.
Conflicts of interest: HK has received honoraria as a speaker and for consultancy from AbbVie, Boehringer Ingelheim, Eisai, Hisamitsu Pharmaceutical, Janssen, Kaken, Kyorin, Kyowa Kirin, Maruho, Meiji Seika Pharma, Mitsubishi-Tanabe, Nihon Pharmaceutical, Novartis, Sanofi, Taiho, and Torii. HF has received honoraria or fees for serving on advisory boards, as a speaker and as a consultant, as well as grants as an investigator from AbbVie, Amgen, Boehringer Ingelheim, Celgene, Chugai Pharmaceutical, Eisai, Eli Lilly, Janssen, Japan Blood Products Organization, JMEC, Kaken, Kyorin, Kyowa Kirin, LEO Pharma, Maruho, Mitsubishi-Tanabe, Nihon Pharmaceutical, Novartis, Sanofi, Sun Pharma, Taiho, Torii, UCB, and Ushio. HT reported no conflicts of interest. TT has received honoraria for speaker and consultancy from AbbVie, Boehringer Ingelheim, Eisai, Janssen, Eli Lilly, Kaken, Kyorin, Maruho, Mitubishi-Tanabe, Nihon Pharmaceutical, Novartis, Sanofi, Sun Pharma, and Taiho.

\section{REFERENCES}

1. Zouboulis CC, Desai N, Emtestam L, Hunger RE, Ioannides D, Juhász I, et al. European S1 guideline for the treatment of hidradenitis suppurativa/acne inversa. J Eur Acad Dermatol Venereol 2015; 29: 619-644.

2. Onderdijk AJ, van der Zee $H$ H, Esmann S, Lophaven S, Dufour DN, Jemec GB, Boer J. Depression in patients with hidradenitis suppurativa. J Eur Acad Dermatol Venereol 2013; 27: 473-478.

3. Alavi A, Anooshirvani N, Kim WB, Coutts P, Sibbald RG. Quality-of-life impairment in patients with hidradenitis suppurativa: a Canadian study. Am J Clin Dermatol 2015; 16: 61-65.

4. Hayama K, Fujita H, Hashimoto T, Terui T. Japanese HS Research Group. Questionnaire-based epidemiological study of hidradenitis suppurativa in Japan revealing characteristics different from those in Western countries. J Dermatol 2020; 47: 743-748.

5. Ware JE Jr, Kosinski M, Gandek B, Aaronson NK, Apolone $G$, Bech P, et al. The factor structure of the SF-36 Health Survey in 10 countries: results from the IQOLA Project. International Quality of Life Assessment. J Clin Epidemiol 1998; 51: 1159-1165.

6. Sartorius K, Emtestam L, Jemec GB, Lapins J. Objective scoring of hidradenitis suppurativa reflecting the role of tobacco smoking and obesity. Br J Dermatol 2009; 161: 831-839.

7. Fukuhara S, Bito S, Green J, Hsiao A, Kurokawa K. Translation, adaptation, and validation of the SF-36 Health Survey for use in Japan. J Clin Epidemiol 1998; 51: 1037-1044.

8. Fukuhara S, Suzukamo Y. Manual of SF-36v2 Japanese version. Kyoto: iHope International Inc.; 2004, 2015.

9. Hayama K, Fujita H, Iwatsuki K, Terui T. Improved quality of life of patients with generalized pustular psoriasis in Japan: a cross-sectional survey. J Dermatol 2021; 48: 203-206.

10. Montero-Vilchez T, Diaz-Calvillo P, Rodriguez-Pozo JA, Cuenca-Barrales C, Martinez-Lopez A, Arias-Santiago S, Molina-Leyva A. The burden of hidradenitis suppurativa signs and symptoms in quality of life: systematic review and metaanalysis. Int J Environ Res Public Health 2021; 18: 6709.

11. Krajewski PK, Matusiak $Ł$, von Stebut E, Schultheis $M$, Kirschner U, Nikolakis G, Szepietowski JC. Quality-of-life impairment among patients with hidradenitis suppurativa: a cross-sectional study of 1795 patients. Life (Basel) 2021; $11: 34$.

12. Jørgensen AR, Holm JG, Ghazanfar MN, Yao Y, Ring HC, Thomsen SF. Factors affecting quality of life in patients with hidradenitis suppurativa. Arch Dermatol Res 2020; 312: 427-436.

13. Itakura A, Tani $Y$, Kaneko N, Hide M. Impact of chronic urticaria on quality of life and work in Japan: Results of a real-world study. J Dermatol 2018; 45: 963-970.

14. Gotesman RD, Choi C, Alavi A. Hidradenitis suppurativa in East and Southeast Asian populations: a systematic review and meta-analysis. Int J Dermatol 2021; 60: e433-e439. 